\title{
MiR-34a suppresses HNSCC growth through modulating cell cycle arrest and senescence
}

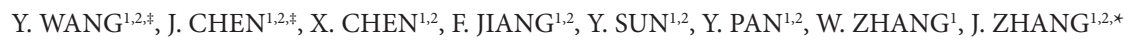 \\ ${ }^{1}$ The State Key Laboratory Breeding Base of Basic Science of Stomatology (Hubei_MOST) \& Key Laboratory of Oral Biomedicine Ministry of \\ Education, School \& Hospital of Stomatology, Wuhan University, Wuhan, China; ${ }^{2}$ Oral Histopathology Department, School and Hospital of \\ Stomatology, Wuhan University, Wuhan, China \\ *Correspondence: jiali_zhang@whu.edu.cn \\ ${ }^{*}$ Contributed equally to this work.
}

Received August 11, 2016 / Accepted January 20, 2017

\begin{abstract}
MiR-34a acts as a tumor suppressor in various malignancies. In HNSCC, the role of miR-34a in proliferation has not been fully elaborated and the target genes are still bind. Here, we addressed that forced miR-34a expression induced cell cycle arrest and senescence. Hypoxia/HIF1a was found to negatively correlate to the expression of miR-34a in HNSCC tissues and partially reverse miR-34a-imposed cell senescence through suppressing miR-34a expression. In order to screen the possible target genes of miR-34a in HNSCC, the differential genes mediated by miR-34a were screened by mRNA microarray. There were 91 genes co-down regulated in two cell lines, which were closely associated with MAPK, ErbB and p53 pathways. Genes, including FUT1, AXL, and MAP2K1 were finally identified as the novel targets of miR-34a by qPCR and luciferase assay. These findings indicate that miR-34a plays an essential role in suppressing HNSCC growth through inducing cell cycle arrest and senescence, by targeting proliferation-associated genes.
\end{abstract}

Key words: head and neck squamous cell carcinoma, miR-34a, cell proliferation, senescence

Head and neck squamous cell carcinoma (HNSCC) is an aggressive epithelial neoplasm, which accounts for upwards of 90 percent of head and neck malignancies [1]. The initiation and progression of HNSCC is a multi-step and long-term process which is attributed to accumulation of genetic and epigenetic alterations [2]. In the last few decades, researchers have found that in HNSCC, miRNAs worked as biomarkers for diagnosis and prognosis, and mediate complex biological processes by interactions with various oncogenes or anti-tumor genes [3].

MicroRNAs, a class of small non-coding RNAs, are 22-24 nucleotides in length. It degrades or silences genes expression post-transcriptionally by fully or partially base-pairing to the complementary sequences in the 3'UTR region of target mRNA. MiR-34a, the most popular member of miR-34 family, has become a novelty tumor-suppressor in diverse malignancies [4]. It is reported to participate in multiple anti-tumor biological processes, such as induction of cell-cycle arrest, senescence, and apoptosis, and reduction of metastasis, angiogenesis and chemo-resistance through regulating different targets [5-11]. Notably, miR-34a is identified as an essential tumor growth suppressor, because it is a significant downstream target of p53 after DNA damage. P53 activation directly up-regulates miR-34 leading to the induction of the apoptosis, cell cycle arrest, or senescence. Further researches indicate a group of genes, such as SIRT1, Bcl-2, CDK4, cyclin D1 and c-myc, have been identified as direct targets of miR-34a in some solid tumors [12, 13]. Moreover, pathways like Wnt, Notch or Akt signaling, are ascertained to be involved in miR34 a mediated cell growth suppressing $[14,15]$. It indicates that multiple target genes and molecular signaling are mediated by miR-34a and may vary in different cancer types $[16,17]$, because of different pathological and cellular environment.

In HNSCC, the reduction of miR-34a was detected in cell lines and tissues which was associated with cell proliferation, angiogenesis and metastasis $[6,18]$. The underlying mechanism of miR-34a in suppressing HNSCC development remains not fully clarified, for few target genes and signaling pathways modulated by miR-34a in HNSCC have been identified. As a result, investigating the changes of cell cycle and cell senescence mediated by miR-34a, and exploring the potential 
target genes of miR-34a in HNSCC is essential to give further understanding of the anti-tumor effect of miR-34a on cell proliferation. Here, we identify miR-34a suppresses HNSCC growth through inducing cell cycle arrest and senescence. Several genes, such as FUT1, AXL, and MAP2K1, directly regulated by miR-34a, might be important in miR-34a modulating tumor repression.

\section{Materials and methods}

Cell lines and tumor samples. UM-SCC-23 derived from larynx was provided by University of Michigan as a gift from Dr.Thomas E. Carey. Fadu cell line derived from a pharyngeal carcinoma and the HEK-293T embryonic kidney cell line were obtained from the American Type Culture Collection (ATCC, Manassas, VA, USA). Fadu and UM-SCC-23 cells were cultured respectively in DMEM medium supplemented with $10 \%$ FBS, 1000 units $/ \mathrm{mL}$ penicillin and $0.1 \mathrm{mg} / \mathrm{ml}$ streptomycin. HEK-293T cells were cultured in RM1640 containing 10\% FBS. When exposed to hypoxia, cells were cultured with $1 \%$ $\mathrm{O}_{2}, 5 \% \mathrm{CO}_{2}, 94 \% \mathrm{~N}_{2}$, and at $37^{\circ} \mathrm{C}$.

39 cases of fresh primary HNSCC samples and the matched adjacent normal epithelial tissues confirmed by pathologists were collected during surgery at the Stomatology Hospital of Wuhan University during 2010-2015. None of the patients received chemotherapy or radiation therapy before surgery. Patients were verified HPV-16 negative upon PCR detection of HPV16. Tissue samples were immediately treated with RNA safe solution (Promoter biotechnology, Wuhan, China) and stored at $-80^{\circ} \mathrm{C}$ before use. The Chinese Ethic Committee approval was received for the examination of patient samples.

Quantitative reverse-transcription PCR (qRT-PCR). Total RNA was isolated from the HNSCC fresh specimens or the cultured cell lines using the mirVana miRNA isolation kit (Ambion, Taxas, USA). To quantify the expression level of miR-34a, TaqMan microRNA assay specific for miR-34a (Applied Biosystems, Carlsband, CA) was used. For mRNA detection, RT was performed following the method of the Primescript RT regent Kit with gDNA Eraser (Takara, Dalian, Japan). qPCR was performed according to the protocol of All-in-One ${ }^{\text {tw }}$ qPCR Mix (Genecopia, Guangzhou, CHINA). The primer sets were provided in the supplementary datal.

Plasmids and stable transfection. HEK-293T cells at the density of $4.0 \times 10^{6}$ were seeded in a $10 \mathrm{~cm}$ cell culture plate and incubated overnight. The next day, a cocktail, made of the lentivirus expression vectors (pCDH-EF1-copGFP-pre-miR-34a or pCDH-EF1-copGFP-control) and pPACK Packaging Plasmid Mix (Systems Biosciences, California, USA) together with the lipofectamine 2000 (Invitrogen, Carlsad, CA) dissolved in the serum-free OPTI-MEM was added to HEK-293T cells. After $48 \mathrm{~h}$ hours, the lentiviral particle solution was harvested and transfected the target cells with polybrene. Fluorescence sorting was used to select transfected cells with GFP expression.

MTT assay. MTT assay was performed by MTT Cell Proliferation and Cytotoxicity Assay Kit (Beyotime Biotechnology,
Jiangsu, China) according to the manufacturer's protocol. In brief, cells were plated in 96 -well plates at a density of $8 \times 10^{2}$ cells per well and incubated in DMEM supplemented with $10 \%$ FBS. At day 1, 2, 4, 6 and 8, cells were treated with 10ul MTT solution per well in the medium, respectively and incubated for $4 \mathrm{~h}$. After that, cells were treated with 100ul formazan and incubated until the formazan was dissolved. The absorbance of each well at $570 \mathrm{~nm}$ was detected by a microplate reader. Experiments were repeated $\geq 3$ times and data were presented as the mean $\pm \mathrm{SD}$.

Flow cytometry assay. $4 \times 10^{5}$ cells per well were seeded into 6 -well plates. When the confluence reached $60 \%$, cells were synchronized by serum starvation for $24 \mathrm{~h}$, and re-entered into the cell cycle by an exchange of a medium with $10 \%$ FBS DMEM for $24 \mathrm{~h}$. Then cells were harvested by trypsinization and fixed with $75 \%$ ethanol at $4{ }^{\circ} \mathrm{C}$ overnight. After washing with cold PBS, cells were incubated with RNase A at $37^{\circ} \mathrm{C}$ for $30 \mathrm{~min}$ and then stained with propidium iodide (PI). The cell cycle status was measured by flow cytometry. The data were analyzed by ModFit software.

SA- $\beta$-Gal Staining. $\beta$-Gal staining was performed by using a Senescence $\beta$-Galactosidase Staining Kit (Beyotime Biotechnology, Jiangsu, China) according to the manufacturer's protocol. $1.5 \times 10^{5}$ cells were incubated in 6-well plates with round cover slips in DMEM supplemented with $10 \%$ FBS. After incubation for certain time, cells were fixed with fixing solution for $15 \mathrm{~min}$ at RT and washed with phosphate-buffered saline. Then, cells were immersed with SA- $\beta$-Gal solution at 37 ${ }^{\circ} \mathrm{C}$ in a $\mathrm{CO}_{2}$-free atmosphere until SA- $\beta$-gal staining became visible. The cells were stained with Eosin and covered with round coverslips. Through a microscope, the flattened and blue-staining cells were scored as senescent. The senescent rate was calculated as the percentage of the senescent cells on all cells observed per high-power field. Each group cells were counted from 10 random microscopic fields and calculated the mean values.

MRNA microarray analysis. Total RNA was extracted from UM-SCC-23 and Fadu cell lines transfected with premiR-34a or pre-miR-control using Trizol (Invitrogen, Carlsad, CA) according to the manufacturer's protocol and purified by NucleoSpin ${ }^{\circledast}$ RNA clean-up kit (MACHEREY-NAGEL, Germany). The 22k human Genome Array Gene chips using two-channel technology were constructed at Capital BioCorporation. In brief, $5 \mu \mathrm{g}$ of total RNA was used to synthesize double-stranded cDNA templates and cRNA was obtained using T7 Enzyme Mix. The products of reverse transcription of cRNA were KLENOW enzyme-labeled and then coupled to the Cy5-dCTP or Cy3-dCTP fluorescent dye (GE Healthcare, United States). The labeled-cDNA microarray was dissolved into the hybridization solution and incubated at $42{ }^{\circ} \mathrm{C}$ overnight. After washing with $0.2 \%$ SDS, $2 \times \mathrm{SSC}$ buffer, it could be scanned with a confocal LuxScan scanner.

Bioinformatics analysis. Targetscan (http://www.targetscan.org/) and miRbase (http://www.mirbase.org/) were used for predicting the target genes of miR-34a. Pathway analysis 
was performed based on the KEGG database (http://www. genome.jp/kegg/). With a P-value $<0.05$, the enriched pathways were determined.

Cloning of 3'UTR. The full or partial length of 3'UTRs in the candidate genes carrying the putative miR-34a binding sites were amplified by PCR using CDNA from UM-SCC-23 cell line. The 3'UTRs were cloned into the pSiCheck ${ }^{\mathrm{m}}-2$ vector (Promega, Madison, WI, USA) and then verified by sequencing. Point mutations of seed matching sequences were performed with the Quick Change Mutagenesis kit (Stratagene, United States). Oligonucleotides used for cloning and mutagenesis were provided in supplementary data 2 and 3.

Luciferase assays. Fadu cells were seeded in 6-well plat at the density of $5.0 \times 10^{4}$ and co-transfected with $40 \mathrm{ng}$ the $\mathrm{pSiCheck}^{\mathrm{m}}$-2 luciferase vector (carrying wild-type or mutated 3'UTR sequences), 2 ug miR-34a or miR-control, using Lipofectamine 2000 (Invitrogen, Carlsad, CA). After 48h, the cells were treated with lysis buffer and the reporter activities were detected in three replicates with the Dual Luciferase Reporter assay system (Promega, Madison, Wisconsin, USA) according to manufacturer's method.

Statistical analysis. All statistical analyses were performed using GraphPad Prism5 software system. All data were presented as the mean \pm SEM; groups were compared using two tailed Student's t-test. P values $<0.05$ were considered statistically significant.

\section{Results}

MiR-34a suppresses proliferation and induces cell cycle arrest in HNSCC cell lines. To verify whether miR-34a was important in suppressing tumor growth, miR-34a was stably over-expressed in Fadu (280.5-fold increase) and UM-SCC-23 (258.2-fold increase) cells by transfecting with pre-miR-34a recombinant lentiviral vector (Figure 1A). The cell proliferation rate was assessed by MTT assay at 1-, 2-, 4-, 6-, and 8 day. The result showed that the aberrant expression of miR-34a led to cell growth reduction in the two HNSCC cell lines (Figure $1 \mathrm{~B}, p<0.05)$.

To further ascertain miR-34a-mediated proliferation suppression, cell-cycle distribution was detected using flow cytometry assay. Compared with the wide type and control cells, the miR-34a over-expression cells exhibited an about 1.6 to 2.0 times increase in the percentage of cell at G0/G1 phase, along with a decreased cell percentage at the $S$ phase (Figure 1C). The result showed that forced miR-34a expression in UM-SCC-23 and Fadu cell lines induced cell cycle arrest at $\mathrm{G0} / \mathrm{G} 1$ phase.

MiR-34a induces cellular senescence. In order to observe whether the introduction of miR-34a into HNSCC cells induces senescence, the percentage of positive SA- $\beta$-Galexpressing cells were calculated. The result showed that the mean percentage of SA- $\beta$-gal-positive cells increased by 4.41 times (UM-SCC-23-miR-34a) and 8.18 times (Fadu-miR-34a) in miR-34a over-expression cell lines than that in control cell lines (Figure 1D, $\mathrm{p}<0.0001$, Supplementary data 4).

Hypoxia partially reduces miR-34a-imposed cell senescence through suppressing miR-34a in HNSCC cell line. To explore the effect of hypoxia on miR-34a induced cell senescence, the UM-SCC-23 cells with or without miR-34a overexpression were exposed to hypoxia condition at different time point. Under the hypoxia condition, the mean percentage of senescent cells in UM-SCC-23-miR-34a cells was declined with the prolonging of exposure time (Figure $2 \mathrm{~A}, p<0.001$ ). Although, compared with control cells, miR-34a over-expression cells remained much higher cell senescence rate at the same hypoxic time point (Figure 2A and Supplementary data 5).

To identify whether hypoxia suppresses miR-34a expression, the relative level of miR-34a in UM-SCC- 23 cells was investigated. It showed that the miR-34a expression was downregulated under hypoxic environment in a time-dependent manner. (Figure $2 \mathrm{~B}, p<0.05$ ).

The expression of miR-34a is markedly decreased in the HNSCC tissues with increased HIF1- $\alpha$ level. To detect the relationship between tumor hypoxia micro-environment and miR-34a levels in tissue, the expressions of miR-34a and HIF1- $\alpha$ in HNSCC specimen and adjacent normal epithelial tissues were analyzed by using quantitative RT-PCR. Compared with the adjacent normal epithelial, 15 of 39 HNSCC samples showed increased HIF1- $\alpha$ expression ( $\log 2$ foldchange $\geq 0.4), 5$ samples revealed a reduction of HIF $1-\alpha(\log 2$ fold-change $\leq-0.4)$ and 19 samples showed no significant difference $(-0.4<\log 2$ fold-change $<0.4)$ (Figure $3 \mathrm{~A})$. About 2 -fold reduction of miR-34a expression was found in 12 of $15(80 \%)$ HIF1- $\alpha$ elevated samples (Figure 3B). Although, the reversed expression trend between miR-34a and HIF1- $\alpha$ was displayed in all 39 tumor tissues, the statistical negative correlation was not significant (Figure 3C, $\mathrm{r}^{2}=0.0335, p=0.2648$ ).

Profile the miR-34a downstream genes by microarray and bioinformatics analysis. To screen the possible downstream genes regulated by miR-34a at mRNA levels in HNSCC cells, the genome-wide mRNA expression of HNSCC cells with or without miR-34a over-expression were performed by two-channel mRNA microarray analysis. Compared with the control, 1578 genes and 1900 genes were detected down-regulated respectively in Fadu-miR-34a and UM-SCC23-miR-34a cells, when the differential mRNAs with a $\log 2$ (fold-change) $<-0.6$ were considered. To get the intersection, there were 91 genes co-down-regulated in Fadu and UMSCC-23 on account of miR-34a over-expression. The heat map of the co-down-regulated genes was generated from the microarray data reflecting the expression values (Figure 4A).

The pathway analysis based on the KEGG pathway database was manipulated on the 91 co-down-regulated genes. The KEGG enrichment analysis revealed that these genes were strikingly involved in MAPK signaling pathway, ErbB signaling pathway and p53 pathway, and moderately regulated the biological process of apoptosis, methionine metabolism, $\mathrm{T}$ cell receptor signaling pathway and so on (Figure 4B). 

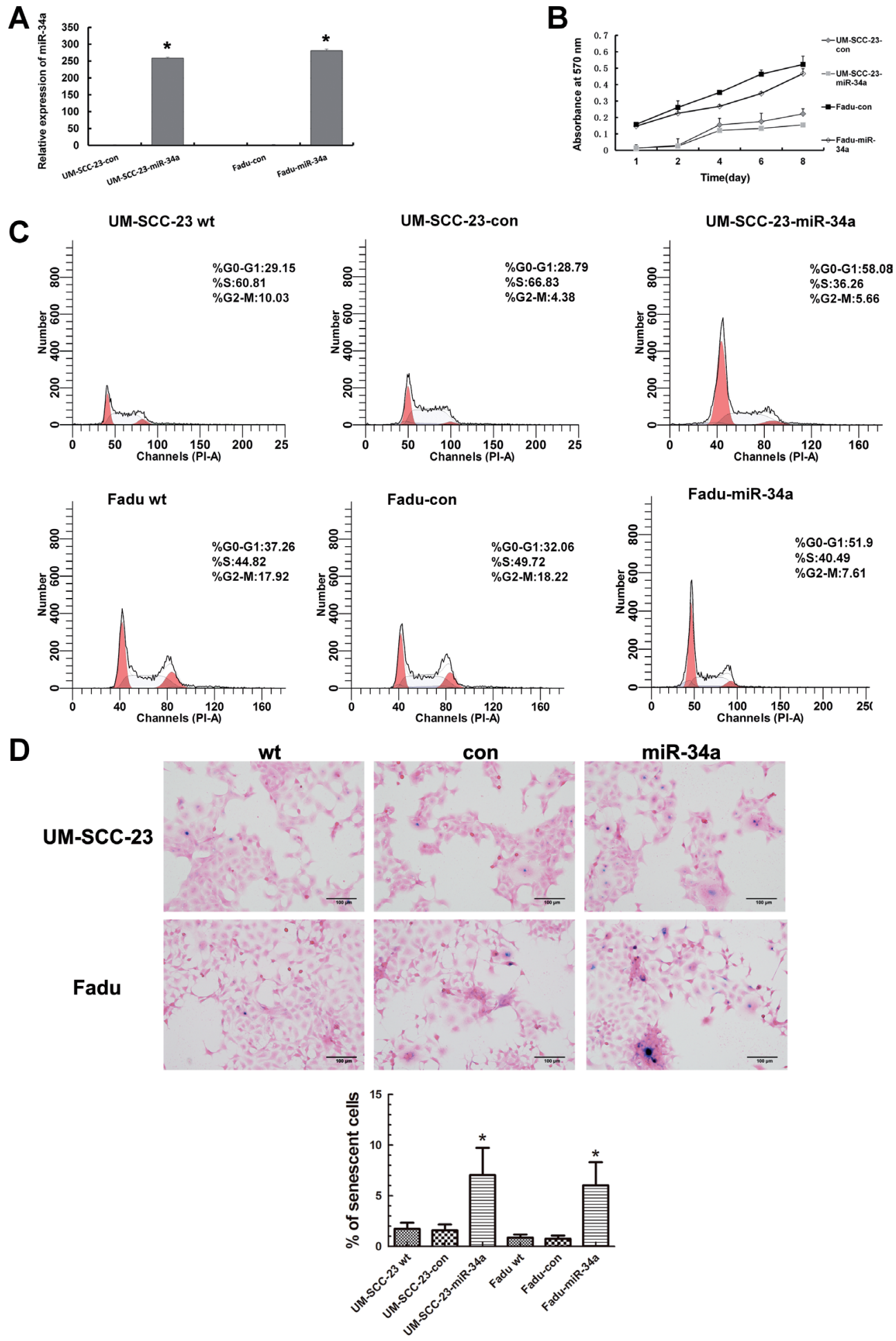

Figure 1. MiR-34a suppressed the proliferation and promoted senescence of HNSCC cell lines in vitro. (A) The relative expressions of miR-34a in HNSCC cell lines. Cells transfected with pre-miR-34a recombinant lentiviral vector showed a significant increase in relative miR-34a level. (B) Cell proliferation ability was reduced in the cells over-expressed miR-34a determined by MTT. Their optical density of viable cells was assayed and measured at $490 \mathrm{~nm}$ (OD). Based on the mean absorbance, the proliferation curves were drawn at each time-point. (C) Cell cycle distribution of HNSCC cell lines measured by flow cytometry. MiR-34a overexpression causes cell cycle arrest in G0/G1 phase (the cell percentage increased at Channel 50). The representative cell cycle distributions were shown in the photograph, which contained the average percentages of G0/G1, $S$ and G2/M phases. (D) More senescent cells determined by Senescence $\beta$-Galactosidase Staining were detected in the UM-SCC-23-miR-34a and Fadu-miR-34a cell lines than that in wide-type and control cell lines. Blue precipitation in the cytoplasm was observed in the senescent cells. Images are magnified 200x. 
MAP2K1, AXL, FUT1 and AREG are the direct targets of miR-34a in HNSCC. Using in-silico miRNA targets identification tools, miRbase and Targetscan4.2, twenty-five of the ninety-one genes were identified as potential targets of miR-34a (Figure 5A). Considering the mRNA fold-change, mRNA abundance (the fluorescence intensity $\geq 1000$ ) and gene function associated with tumorigenesis, 11 of the 25 genes were selected to be further analyzed, which were revealed in Figure 5A.

To verify whether those 11 genes were down regulated by miR-34a in HNSCC cells, the mRNA expression level was detected by qRT-PCR. The result indicated that the mRNA levels of 8 genes were reduced about 1.4 -fold to 4.7 -fold in cells with ectopic miR-34a expression, compared with control (Figure 5B).

To further confirm whether the 8 candidate genes were directly targeted by miR-34a, dual reporter assay was performed, respectively. AREG was validated to be a direct target gene involving in metastasis in our previous study [6]. The

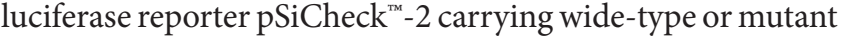
3'UTR sequences of the other 7 genes were constructed and then co-transfected with either miR-34a or miR-control in Fadu cell line (Figure 6A). The luciferase reporter activity of pSiCheck ${ }^{\mathrm{m}}$-2-AXL-3'UTR, pSiCheck ${ }^{\mathrm{m}}$-2-FUT1-3'UTR

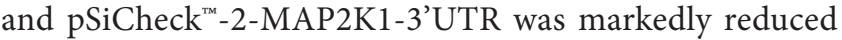
by forced expression of miR-34a compared with the control (2.43-fold decrease, 2.08-fold decrease and 4.78-fold decrease, respectively), whereas no reporter activity were found when their target sites were mutated (Figure 6B-C). According to Targetscan, the 3'UTR of FUT1 mRNA has two predicted binding sites of miR-34a. To identify the two binding sites respectively, reporter vector $\mathrm{pSiCheck}^{\mathrm{Tw}}-2$ carrying point mutations at the two putative seed binding sequences were respectively constructed. The result demonstrated that when mutagenesis at the position 1567-1574 of FUT1 3' UTR (mut1), miR-34a had a minimal effect on reporter activity; while when mutagenesis at the position 1641-1647 (mut2), the significant reduction of reporter activity was still detected. The result indicated that position 1567-1574 of FUT1 3' UTR (mut1) is the specific target sequence of miR-34a.

In addition, in order to investigate the effect of hypoxia on miR-34a target genes, the mRNA expressions of FUT1, AXL, AREG and MAP2K1 were evaluated after hypoxia for 24 hours. The result showed that the relative mRNA expression levels of MAPK2 and FUT1 in HNSCC cell lines were significantly induced, when miR-34a was suppressed by hypoxia, while AREG and AXL remained unchanged (Supplementary data 6).

\section{Discussion}

Head and neck cancer represent an intramurally heterogeneous cohort of malignant tumors which originate from oral cavity, oropharynx, hypopharynx, or larynx [6]. This disease can significantly interfere with psychological health and life quality of the patients $[19,20]$. Investigations of potential

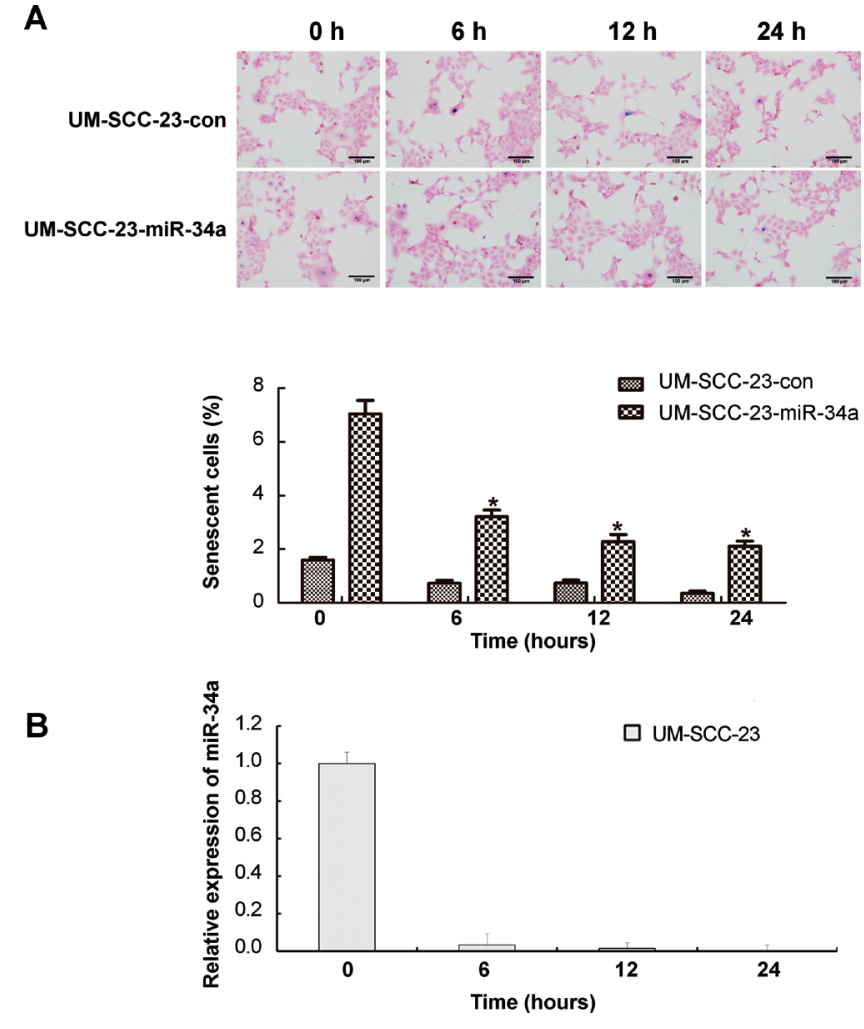

Figure 2. The expression of miR-34a and senescence of HNSCC cells under hypoxia. (A) The senescence of cells over-expressed miR-34a or control under hypoxia at time points 6,12 , and 24 hours was evaluated, respectively. Compared with control cells, the senescent rate of cells over-expressed miR-34a was dramatically decreased under hypoxia in a time-dependent manner. Images are magnified 200×. (B) The relative expression of miR34 a was dramatically repressed by hypoxia in a time-dependent manner.

biomarkers and therapeutic targets have become focal point in the molecular oncology of HNSCC. Numerous evidences have indicated that miRNAs, involved in the initiation and progression of tumors, possess an anti-tumor activity in HNSCC.

MiR-34a is one of the best-studied miRNAs associated with malignancies. It is located at the chromosome lp36 locus, where is susceptive of chromosomal aberration and induces multiple molecular events thereby disturbing the dynamic cell balance of proliferation, apoptosis, senescence and metastasis [21-25]. In HNSCC, the decreased expression of miR-34a was observed previously [6]. The present study further demonstrated that ectopic miR-34a expression retarded proliferation and induced G0/G1 cell cycle arrest in HNSCC cell lines, which was consistent with observations in colon cancer, hepatocellular cancer and non-small cell lung cancer [26-28]. On the other hand, miR-34a was reported to promote cell senescence in vascular smooth muscle cells, colon cancer and hepatocellular cancer [29-31]. Here, we provided evidence that miR-34 promoted cell senescence in HNSCC. Although the direct target of miR-34a involved in senescence was still ambiguous in HNSCC, based on 

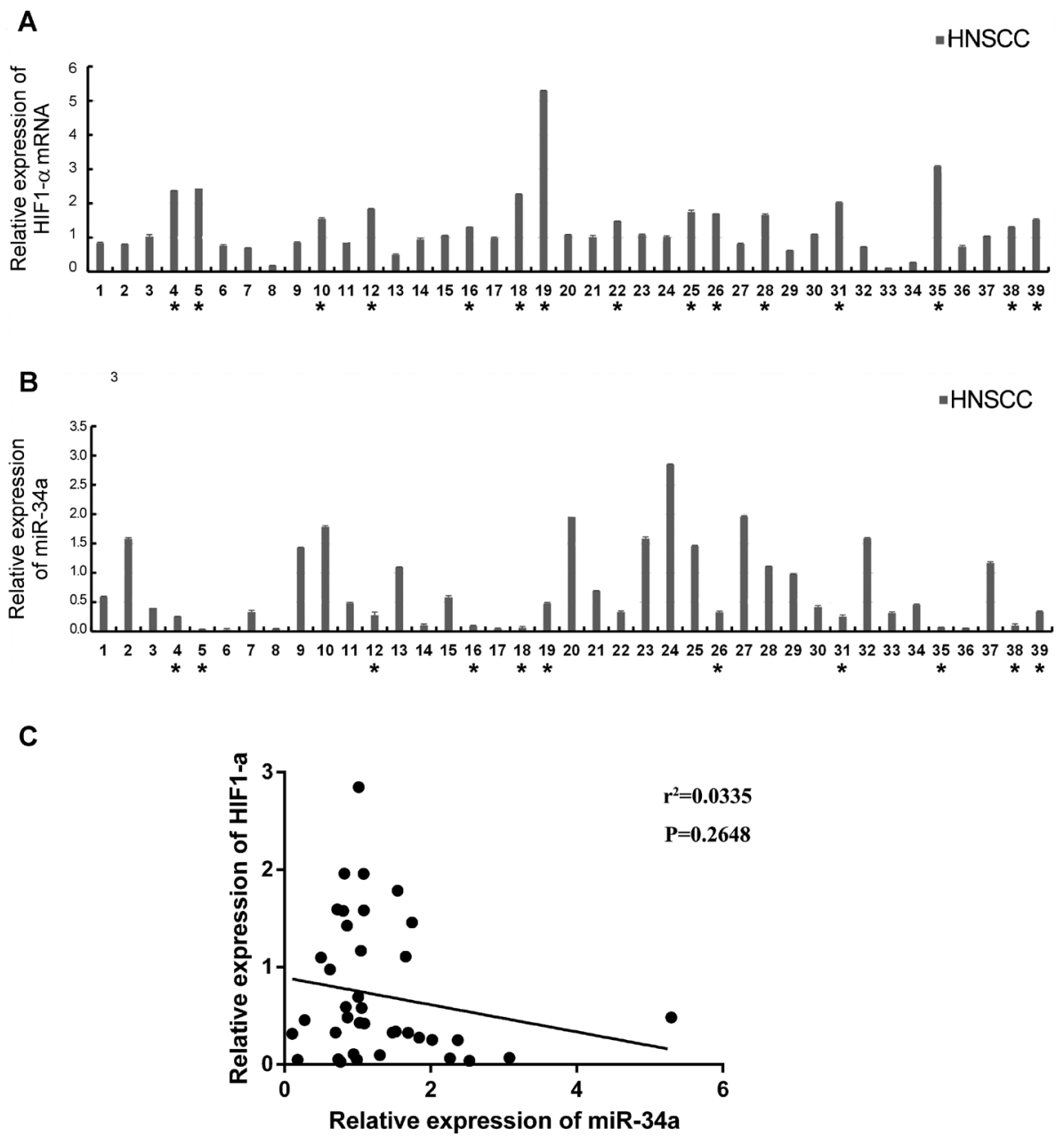

Figure 3. The expressions of HIF1- $\alpha$ mRNA and miR-34a in HNSCC samples. (A) The black asterisks represented the HNSCC samples with increased HIF1- $\alpha$ expression (log2 fold-change $\geq 0.4$ ), compared with adjacent normal epithelia. (B) The black squares indicated that in HIF1- $\alpha$ elevated samples, $80 \%$ of which showed 2-fold reduction of miR-34a expression. (C) General correlation between miR-34a and HIF1- $\alpha$ mRNA relative expression in 40 samples. The statistical negative correlation was not significant $(\mathrm{P}>0.05)$.

our bioinformatics data, several genes and signaling pathways involved in senescence or cell death were significantly downregulated, such as MAPK and P53 signaling pathway, TFE3, and MEK1. Additionally, we observed that hypoxia could suppress miR-34a expression, thereby reducing miR-34a imposed cell senescence. It was suggested that miR-34a, acted as an inhibitor of tumor cell growth through cell cycle arrest and cellular senescence, could constitute a bona fide barrier to progression of HNSCC, whereas intro-tumor hypoxia could impair the binding effect of miR-34a on tumor growth through decreasing its expression. Moreover, the relative mRNA expression levels of MAPK2 and FUT1 in HNSCC cell lines were detected significantly induced by hypoxia, while AREG and AXL remained unchanged. The result might indicate that MAPK2 and FUT1, the potential target genes suppressed by miR-34a, were induced by hypoxia, which might be because that miR-34a was significantly reduces under the hypoxic environment.
To ascertain target genes of miR-34a is of major significance to decipher its molecular functional mechanism in tumor development. Our research identified FUT1, AXL, and MAP2K1 as the direct targets of miR-34a. FUT1, which encodes $\alpha 1$, 2 -fucosyltransferase and transfers a fucose residue to galactose, is of key significance to the biosynthesis of Lewis $\mathrm{Y}$ antigen [32]. Up-regulated Lewis $Y$ has been found in various tumors including breast, ovarian, and colorectal cancer [33-35], leading to tumor growth acceleration and lymph node metastasis through activating ErbB signaling [36]. To our knowledge, FUT1 is for the first time to be demonstrated as the target of miR-34a. The specificity target sequence of FUT1 3'UTR is at the 1567-1574 region, but not the 1641-1647 region. Coincidentally, AREG, another target confirmed in HNSCC, is also tightly associated with ErbB signaling. By binding to the EGFR, AXL, a member of receptor tyrosine kinases [37], stimulates a mix of physiological processes of normal cells 
A
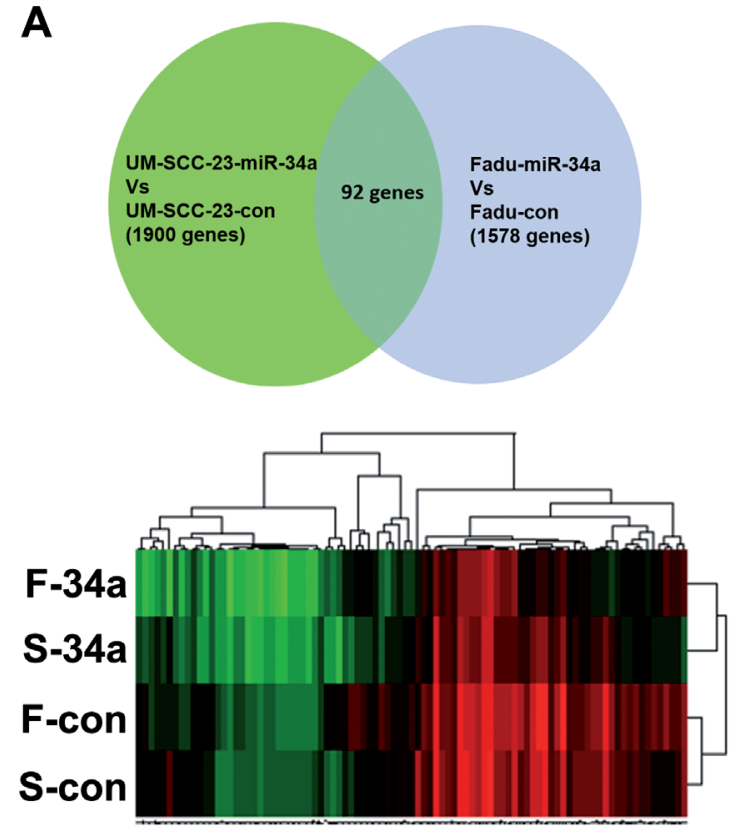

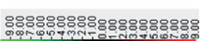

B

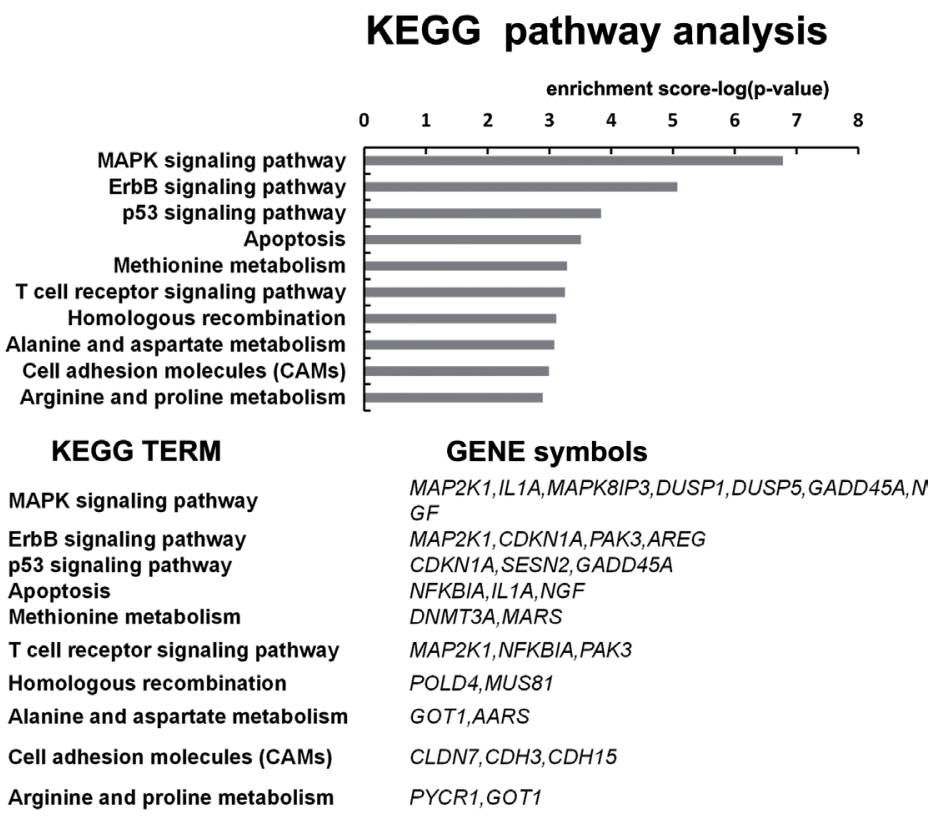

Figure 4. KEGG pathway analysis of co-down regulated genes. (A) Venn diagram of miR-34a mediated changes in genome wide mRNA expression. The overlap field revealed the co-down-regulated genes with $\log 2$ (fold change) $\leq-0.6$. The heat map below generated from the microarray data reflected the co-down-regulated gene expression values in F-34a, S-34a, F-con, S-con cell lines. (B) KEGG pathway analysis for the co-down-regulated genes. The enrichment scores of biological processes were shown as - $\log 2$ ( $p$-value). For each KEGG pathway, the gene symbols of differentially down-regulated genes were given in the table below.
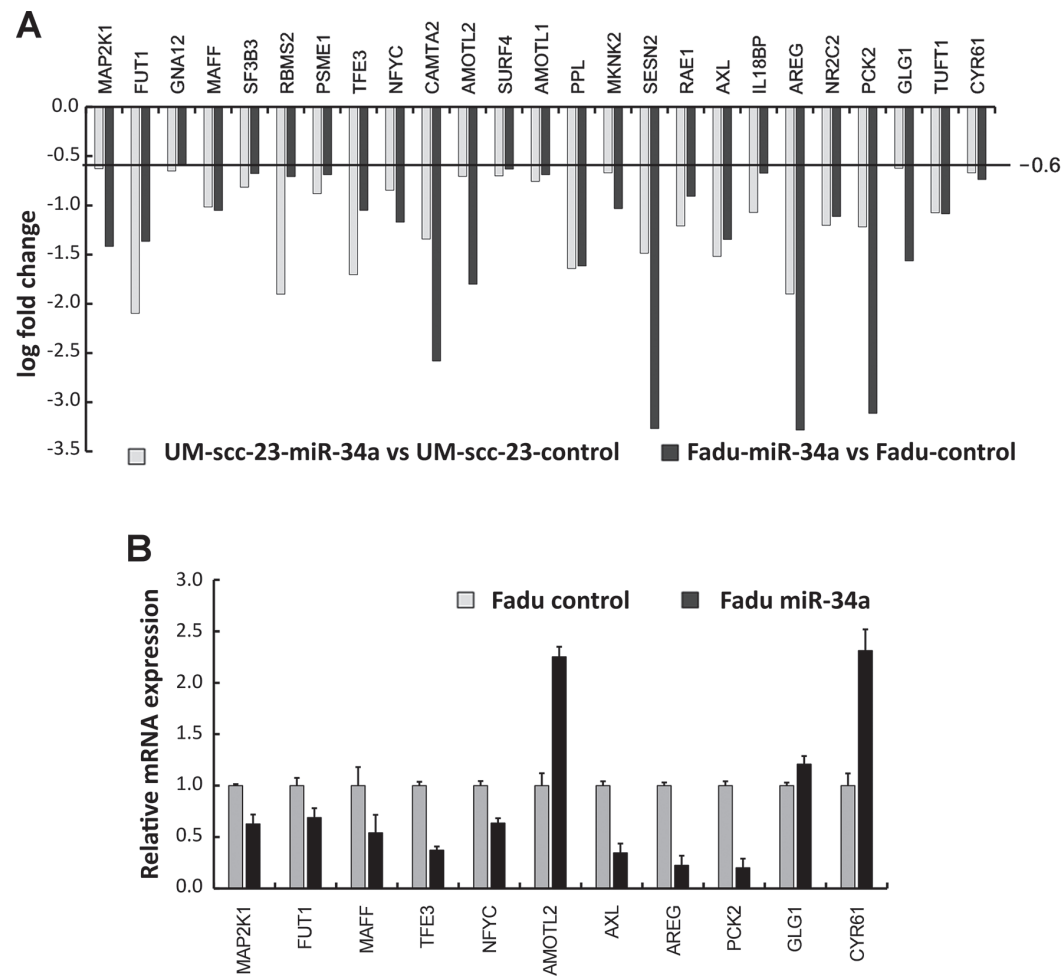

Figure 5. (A) Histogram showing the fold change of 25 co-down-regulated genes with putative target sites of miR-34a after stable transfection of pri-miR34a in Fadu and UM-SCC-23 cells. Log2 (fold change) $\leq-0.6$ were considered. (B) 11 tumor-related genes with mRNA abundance $>1000$ was detected by qRT-PCR. 8 of 11 genes had a general reduction at mRNA level in HNSCC cell lines with aberrant miR-34a expression. 
or cancer cells [38-39]. Studies indicate that the capacity of HNSCC cellular growth, migration, and invasion are dependent on AXL through PI3K/AKT and MAPK signaling [40, 41]. It is well-known miR-34a targets AXL in non-small lung cancer, ovarian cancer, B-cell chronic lymphocytic, breast cancer and colorectal cancer resulting in tumor proliferation [42-44]. In HNSCC, the direct interaction between miR-34a and AXL was evidenced in this study, demonstrating AXL might be a common target of miR-34a in malignant tumors. As the central node in the MAPK signaling cascade (Ras/

A
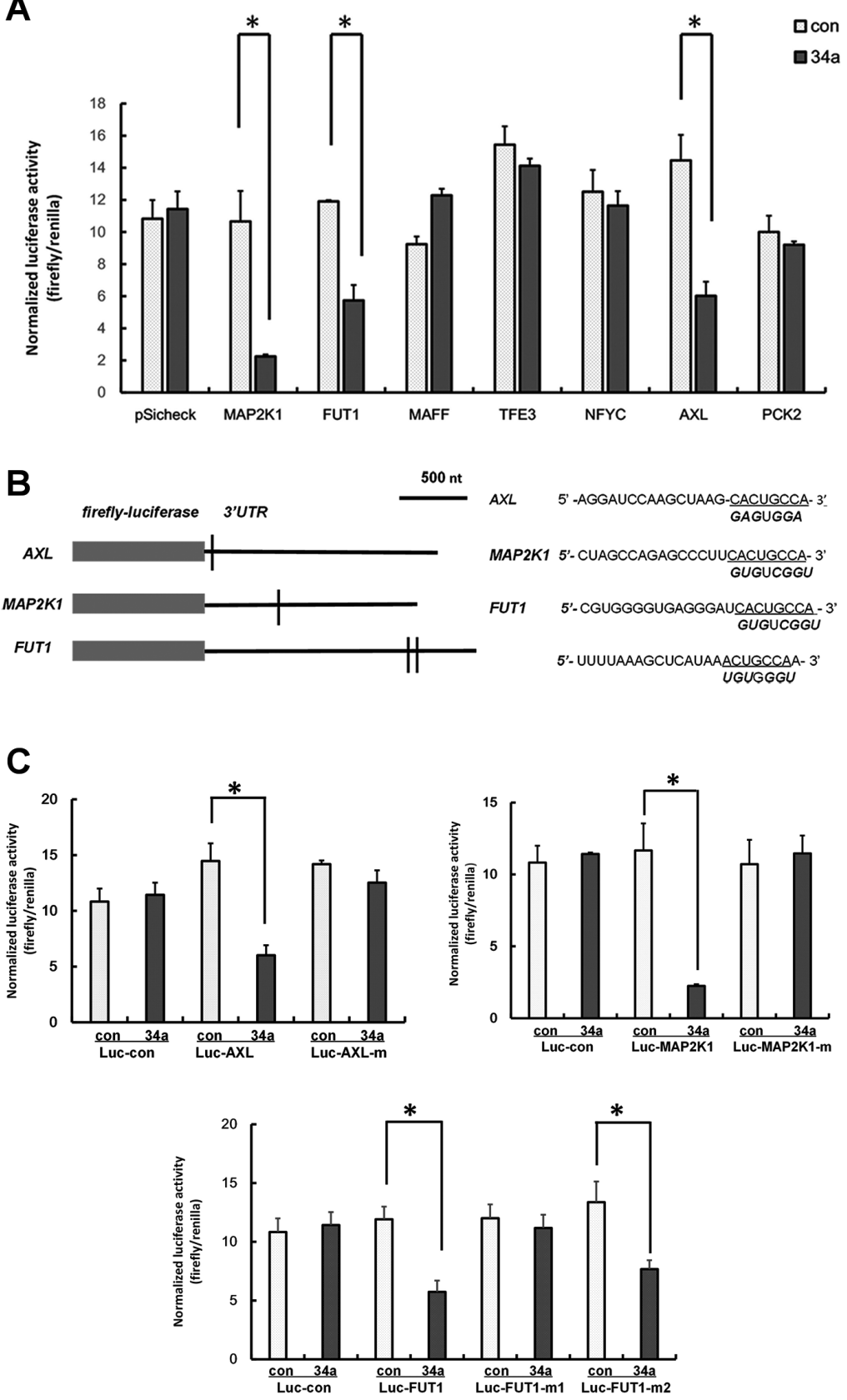

Figure 6. Experimental validation of miR-34a target genes. (A) Dual reporter assays in Fadu cells transfected with pre-miR-34a or control and the indicated 3'-UTR-reporter constructs. The 3'UTR -reporter activity of FUT1, MAP2K1, and AXL were markedly reduced by forced expression of miR-34a compared with the vector control. Data were represented as mean \pm S.D. $(n=3)$. (B) Upper-left schematic depicted miR-34a seed-matching sequences in the 3'UTRs of selected mRNAs. Black vertical bars represented miR-34a seed-matching sites. Upper-Right, the putative miR-34a binding sequence of each target gene were given in the 5' to 3'orientation. The seed-matching sequences were underlined. Below each miR-34a binding site, the mutant seedmatching sequence were shown with exchanged nucleotides high-lighted in bold letters. (C) Luciferase assay with selected 3'UTR reporter constructs and variants with mutant seed-matching sequences. 
Raf/Mek/ERK), MAP2K1 is another newly detected target of miR-34a in HNSCC. It is a dual-specificity tyrosine threonine protein kinase and confers hallmarks of cancer cells such as cell growth, migration and invasion via phosphorylation of ERK1 and ERK2. It has been reported miR-34a targeted MAP2K1 hampering cell proliferation during megakaryocytic differentiation of K562 cells [45]. Thus, it shows that miR-34a may suppress HNSCC proliferation through mainly inhibiting the MAPK and ErbB signaling pathway. In addition, we found that the relative mRNA expression levels of MAPK2 and FUT1 were significantly induced, when miR-34a was suppressed by hypoxia. The result indicates that miR-34a might play an important role in hypoxia induced MAPK2 and FUT1. They induced by hypoxia might be because that miR-34a was significantly reduced under the hypoxic environment.

Gene expression profiling has emerged as an effective approach to study the mechanism of miR-34a in HNSCC development from comprehensive perspective. Among the downstream genes, even though most of them do not carry putative miR-34a binding sites in their 3'UTRs, it was likely to be a secondary consequence of the targets of miR-34a. Furthermore, the bioinformatics analysis showed that these downstream genes consisted of signaling nodes, enzymes and transcription factors, which were enriched in the MAPK, ErbB and P53 signaling pathways. MiR-34a may involve in multiple tumor suppressive pathways by directly or indirectly inhibiting the expression of numerous oncogenes and acts as a molecular switch in a complicated regulatory network.

In HNSCC, besides the inhibition of metastasis with a pivotal target AREG previously reported by our research group, the anti-growth effect of miR-34a via cell cycle arrest and senescence was investigated in the present study. Then 3 novel target genes (FUT1, AXL, and MAP2K1) of miR-34a involved in ErbB and MAPK signaling pathways were identified through mRNA array and luciferase array. It is well known miRNAs post-transcriptionally regulate gene expression by not only mRNA degradation but translational silencing. Future studies combining high-throughput mRNA with proteomics sequencing would further clarify the mechanisms of miR-34a in HNSCC cell proliferation.

Supplementary information is available in the online version of the paper.

Acknowledgements: We thank Mr. Shichun Xiong and Ms. Yuan Li of Wuhan University for technical assistance, Professor Carey of Michigan University for presenting UM-SCC-23 cell line. This project was funded by National Natural Science Foundation of China under the grant nos. [81172571] and [81572664].

\section{References}

[1] THOMPSON L. World Health Organization classification of tumours: pathology and genetics of head and neck tumours. Ear Nose Throat J 2006; 85: 74.
[2] CLOOS J, SPITZ MR, SCHANTZ SP, HSU TC, ZHANG ZF et al. Genetic susceptibility to head and neck squamous cell carcinoma. J Natl Cancer Inst 1996; 88: 530-535. https://doi. org/10.1093/jnci/88.8.530

[3] CHEN D, CABAY RJ, JIN Y, WANG A, LU Y et al. MicroRNA Deregulations in Head and Neck Squamous Cell Carcinomas. J Oral Maxillofac Res 2013; 4: e2-e2. https://doi.org/10.5037/ jomr.2013.4102

[4] LI XJ, REN ZJ, TANG JH. MicroRNA-34a: A potential therapeutic target in human cancer. Cell Death Dis 2014; 5: e1327. https://doi.org/10.1038/cddis.2014.270

[5] KRACIKOVA M, AKIRI G, GEORGE A, SACHIDANANDAM R, AARONSON SA. A threshold mechanism mediates $\mathrm{p} 53$ cell fate decision between growth arrest and apoptosis. Cell Death Differ 2013; 20: 576-588. https://doi.org/10.1038/cdd.2012.155

[6] ZHANG J, WANG Y, CHEN X, ZHOU Y, JIANG F et al. MiR-34a suppresses amphiregulin and tumor metastatic potential of head and neck squamous cell carcinoma (HNSCC). Oncotarget 2015; 6: 7454-7469. https://doi.org/10.18632/ oncotarget. 3148

[7] TAZAWA H, TSUCHIYA N, IZUMIYA M, NAKAGAMA H. Tumor-suppressive miR-34a induces senescence-like growth arrest through modulation of the E2F pathway in human colon cancer cells. Proc Natl Acad Sci U S A 2007; 104: 15472-15477. https://doi.org/10.1073/pnas.0707351104

[8] LIAO H, XIAO Y, HU Y, XIAO Y, YIN Z et al. Methylationinduced silencing of miR-34a enhances chemoresistance by directly upregulating ATG4B-induced autophagy through AMPK/mTOR pathway in prostate cancer. Oncol Rep 2016; 35: 64-72.

[9] SIEMENS H, JACKSTADT R, KALLER M, HERMEKING $\mathrm{H}$. Repression of c-Kit by p53 is mediated by miR-34 and is associated with reduced chemoresistance, migration and stemness. Oncotarget 2013; 4: 1399-1415. https://doi. org/10.18632/oncotarget.1202

[10] LI G, YAO L, ZHANG J, LI X, DANG S et al. Tumor-suppressive microRNA-34a inhibits breast cancer cell migration and invasion via targeting oncogenic TPD52. Tumour Biol 2016; 37: 7481-7491. https://doi.org/10.1007/s13277-015-4623-4

[11] CHENG J, ZHOU L, XIE QF, XIE HY, WEI XY et al. The impact of miR-34a on protein output in hepatocellular carcinoma HepG2 cells. Proteomics 2010; 10: 1557-1572. https:// doi.org/10.1002/pmic.200900646

[12] BOMMER GT, ISABELLE G, YING F, KACZOROWSKI AJ, KUICK R et al. p53-Mediated Activation of miRNA34 Candidate Tumor-Suppressor Genes. Curr Biol 2007; 17: 1298-1307. https://doi.org/10.1016/j.cub.2007.06.068

[13] KALLER M, LIFFERS ST, OELJEKLAUS S, KUHLMANN $\mathrm{K}, \mathrm{ROH} \mathrm{S}$ et al. Genome-wide Characterization of miR-34a Induced Changes in Protein and mRNA Expression by a Combined Pulsed SILAC and Microarray Analysis. Mol Cell Proteomics 2011; 10: M111.010462.

[14] RATHOD SS, RANI SB, KHAN M, MUZUMDAR D, SHIRAS A. Tumor suppressive miRNA-34a suppresses cell proliferation and tumor growth of glioma stem cells by targeting Akt and Wnt signaling pathways. FEBS Open Bio 2014; 4: 485-495. https://doi.org/10.1016/j.fob.2014.05.002 
[15] PARK EY, CHANG E, LEE EJ, LEE HW, KANG HG et al. Targeting of miR-34a-NOTCH1 axis reduced breast cancer stemness and chemoresistance. Cancer Res 2014; 74: 75737582. https://doi.org/10.1158/0008-5472.CAN-14-1140

[16] GHANDADI M, SAHEBKAR A1. MicroRNA-34a and its target genes: Key factors in cancer multidrug resistance. Curr Pharm Des 2016; 22: 933-939. https://doi.org/10.217 4/1381612822666151209153729

[17] AGOSTINI M, KNIGHT RA. miR-34: from bench to bedside. Oncotarget 2014; 5: 872-881. https://doi.org/10.18632/oncotarget. 1825

[18] KUMAR B, YADAV A, LANG J, TEKNOS TN, KUMAR P. Dysregulation of microRNA-34a expression in head and neck squamous cell carcinoma promotes tumor growth and tumor angiogenesis. PLoS One 2012; 7: e37601. https://doi. org/10.1371/journal.pone.0037601

[19] PUGLIANO FA, PICCIRILLO JF, ZEQUEIRA MR, EMAMI B, PEREZ CA et al. Clinical-severity staging system for oropharyngeal cancer: five-year survival rates. Arch Otolaryngol Head Neck Surg 1997; 123: 1118-1124. https://doi. org/10.1001/archotol.1997.01900100094013

[20] PICCIRILLO JF, WELLS CK, SASAKI CT, FEINSTEIN AR. New clinical severity staging system for cancer of the larynx. Five-year survival rates. Ann Otol Rhinol Laryngol 1994; 103: 83-92. https://doi.org/10.1177/000348949410300201

[21] COLE KA, ATTIYEH EF, MOSSE YP, LAQUAGLIA MJ, DISKIN SJ et al. A functional screen identifies miR-34a as a candidate neuroblastoma tumor suppressor gene. Mol Cancer Res 2008; 6: 735-742. https://doi.org/10.1158/1541-7786. MCR-07-2102

[22] HENRICH KO, SCHWAB M, WESTERMANN F. 1p36 tumor suppression--a matter of dosage? Cancer Res 2012; 72: 6079-6088. https://doi.org/10.1158/0008-5472.CAN-12-2230

[23] LI L, YUAN L, LUO J, GAO J, GUO J et al. MiR-34a inhibits proliferation and migration of breast cancer through downregulation of Bcl-2 and SIRT1. Clin Exp Med 2013; 13: 109-117. https://doi.org/10.1007/s10238-012-0186-5

[24] LIU K, HUANG J, XIE M, YU Y, ZHU S et al. MIR34A regulates autophagy and apoptosis by targeting HMGB1 in the retinoblastoma cell. Autophagy 2014; 10: 442-452. https:// doi.org/10.4161/auto.27418

[25] CAO W, YANG W, FAN R, LI H, JIANG J et al. miR-34a regulates cisplatin-induce gastric cancer cell death by modulating PI3K/AKT/survivin pathway. Tumour Biol 2014; 35: 1287-1295. https://doi.org/10.1007/s13277-013-1171-7

[26] BU P, CHEN KY, CHEN JH, WANG L, WALTERS J et al. A microRNA miR-34a-Regulated Bimodal Switch Targets Notch in Colon Cancer Stem Cells. Cell Stem Cell 2013; 12: 602-615. https://doi.org/10.1016/j.stem.2013.03.002

[27] GOUGELET A, SARTOR C, BACHELOT L, GODARD C, MARCHIOL $C$ et al. Antitumour activity of an inhibitor of miR-34a in liver cancer with $\beta$-catenin-mutations. Gut 2016; 65: 1024-1034. https://doi.org/10.1136/gutjnl-2014-308969

[28] GALLARDO E1, NAVARRO A, VIÑOLAS N, MARRADES $\mathrm{RM}, \mathrm{DIAZ} \mathrm{T}$ et al. miR-34a as a prognostic marker of relapse in surgically resected non-small-cell lung cancer. Carcinogenesis 2009; 30: 1903-1909. https://doi.org/10.1093/carcin/bgp219
[29] XU X, CHEN W, MIAO R, ZHOU Y, WANG Z et al. miR-34a induces cellular senescence via modulation of telomerase activity in human hepatocellular carcinoma by targeting FoxM1/c-Myc pathway. Oncotarget 2015; 6: 3988-4004. https://doi.org/10.18632/oncotarget.2905

[30] TAZAWA H, TSUCHIYA N, IZUMIYA M, NAKAGAMA H. Tumor-suppressive miR-34a induces senescence-like growth arrest through modulation of the E2F pathway in human colon cancer cells. Proc Natl Acad Sci U S A 2007; 104: 15472-15477. https://doi.org/10.1073/pnas.0707351104

[31] BADI I, BURBA I, RUGGERI C, ZENI F, BERTOLOTTI M et al. MicroRNA-34a Induces Vascular Smooth Muscle Cells Senescence by SIRT1 Downregulation and Promotes the Expression of Age-Associated Pro-inflammatory Secretory Factors. J Gerontol A Biol Sci Med Sci 2015; 70: 1304-1311. https://doi.org/10.1093/gerona/glu180

[32] ISOZAKI T, RUTH JH, AMIN MA, CAMPBELL PL, TSOU PS et al. Fucosyltransferase 1 mediates angiogenesis, cell adhesion and rheumatoid arthritis synovial tissue fibroblast proliferation. Arthritis Res Ther 2014; 16: R28-R28. https:// doi.org/10.1186/ar4456

[33] GAO N, LIU J, LIU D, HAO Y, YAN L et al. c-Jun transcriptionally regulates alpha 1, 2-fucosyltransferase 1 (FUT1) in ovarian cancer. Biochimie 2014; 107: 286-292. https://doi. org/10.1016/j.biochi.2014.09.015

[34] OKAMURA A, YAZAWA S, MORINAGA N, ASAO T, KUWANO $\mathrm{H}$. Increased thermosensitivity of mouse colorectal carcinoma cells transfected with human FUT1 gene. Cancer Lett 2002; 180: 203-210. https://doi.org/10.1016/S03043835(02)00011-3

[35] CHANG WW, LEE CH, LEE P, LIN J, HSU CW et al. Expression of Globo $\mathrm{H}$ and SSEA3 in breast cancer stem cells and the involvement of fucosyl transferases 1 and 2 in Globo $\mathrm{H}$ synthesis. Proc Natl Acad Sci U S A 2008; 105: 11667-11672. https://doi.org/10.1073/pnas.0804979105

[36] KAWAI S, KATO S, IMAI H, OKADA Y, ISHIOKA C. Suppression of FUT1 attenuates cell proliferation in the HER2overexpressing cancer cell line NCI-N87. Oncol Rep 2012; 29: 13-20.

[37] VERMA A, WARNER SL, VANKAYALAPATI H, BEARSS DJ, SHARMA S. Targeting Axl and Mer kinases in cancer. Mol Cancer Ther 2011; 10: 1763-1773. https://doi. org/10.1158/1535-7163.MCT-11-0116

[38] LINGER RM, KEATING AK, EARP HS, GRAHAM DK. Taking aim at Mer and Axl receptor tyrosine kinases as novel therapeutic targets in solid tumors. Expert Opin Ther Targets 2010; 14: 1073-1090. https://doi.org/10.1517/14728222.2010.515980

[39] FRIDELL YW, JIN Y, QUILLIAM LA, BURCHERT A, MCCLOSKEY P et al. Differential activation of the Ras/ extracellular-signal-regulated protein kinase pathway is responsible for the biological consequences induced by the Axl receptor tyrosine kinase. Mol Cell Biol 1996; 16: 135-145. https://doi.org/10.1128/MCB.16.1.135

[40] ELKABETS M, PAZARENTZOS E, JURIC D, SHENG Q, PELOSSOF RA et al. AXL mediates resistance to PI3Ka inhibition by activating the EGFR/PKC/mTOR Axis in Head and neck and esophageal squamous cell carcinomas. 
Cancer Cell 2015; 27: 533-546. https://doi.org/10.1016/j. ccell.2015.03.010

[41] DUMITRU CA, FECHNER MK, HOFFMANN TK, LANG S, BRANDAU S. A novel p38-MAPK signaling axis modulates neutrophil biology in head and neck cancer. J Leukoc Biol 2012; 91: 591-598. https://doi.org/10.1189/jlb.0411193

[42] BAE SY, HONG JY, LEE HJ, PARK HJ, LEE SK. Targeting the degradation of AXL receptor tyrosine kinase to overcome resistance in gefitinib-resistant non-small cell lung cancer. Oncotarget 2015; 6: 10146-10160. https://doi.org/10.18632/ oncotarget. 3380

[43] VUORILUOTO K, HAUGEN H, KIVILUOTO S, Mpindi JP, Nevo J et al. Vimentin regulates EMT induction by Slug and oncogenic $\mathrm{H}$-Ras and migration by governing Axl expression in breast cancer. Oncogene 2011; 30: 1436-1448. https://doi. org/10.1038/onc.2010.509

[44] PACCEZ JD, VOGELSANG M, PARKER MI, ZERBINI LF. The receptor tyrosine kinase Axl in cancer: Biological functions and therapeutic implications. Int J Cancer 2014; 134: 1024-1033. https://doi.org/10.1002/ijc.28246

[45] ICHIMURA A, RUIKE Y, TERASAWA K, SHIMIZU K, TSUJIMOTO G. MicroRNA-34a inhibits cell proliferation by repressing mitogen-activated protein kinase kinase 1 during megakaryocytic differentiation of K562 cells. Mol Pharmacol 2010; 77: 1016-1024. https://doi.org/10.1124/ $\underline{\text { mol.109.063321 }}$ 
Supplemental Data 1, qPCR oligonucleotides (5' - 3')

HIF1- $\alpha$-F: TCCAAGAAGCCCTAACGTGT

HIF1- $\alpha$-R:TGATCGTCTGGCTGCTGTAA

MAP2K1-F: TTCGCAGAGCGGCTAGGA

MAP2K1-R: CAGCCCGGGATTCCCTTC

FUT1-F: CTAAGGGTGCAAGGGGGAAG

FUT1-R: GCATTTTCTCTGGTCCAAGGC

MAFF-F: GGAAAGCCGGAGAGCAACAACAAA

MAFF-R: TGGACTCCCAAAGTGCTGGGATTA

TFE3-F: TCAGCTGCTCAGCCTGAACTCTTT

TFE3-R: AGTGATATTGGGAGGCTTGAGCGA

NFYC-F: ATCTCATTCCGCCTCCCTCT

NFYC-R: CACCGTTTCCCCTTTTCCCT

MKNK2-F: CCTGGTCCGAGCTACCTCAC

MKNK2-R: GATTTGATTGGGGGACGGGT

CAMTA2-F: GGGACCCCGGGAGAAAACAG

CAMTA2-R: GGCACAAGACAGCCACTCAT

AMOTL2-F: GTGGTTCGCTTTGATCCCGA

AMOTL2-R: GTCAGACACCACAACCTCCG

AXL-F: TCAAGGTGGCTGTGAAGACGATGA

AXL-R: AACCCTGGAAACAGACACCGATGA

AREG-F: TGTCGCTCTT GATACTCGGC

AREG-R: AGGCATTTCACTCACA GGGG

PCK2-F: CGGAGGAAAGCTAGTGCCAG

PCK2-R: TATGGAAGGCGGGGAAGGA

GLG1-F: CAAGGCGTGTCAGTTTGTGG

GLG1-R: AGCTTAGCAGCTTCCCTTGG

CYR61-F: CAGGACTGTGAAGATGCGGT

CYR61-R: GCCTGTAGAAGGGAAACGCT

Supplemental Data 2, List of oligonucleotides used for $3^{\prime}$-UTR cloning $\left(5^{\prime}-3^{\prime}\right)$

AXL-F: CTCGAG ACAACCCTCCACCTGGTACT

AXL-R: GCGGCCGC GGTCCTAGAGAGGCAGAATGC

TFE3-F: CTCGAGTCAGGCCTCACCCCTCCCCT

TFE3-R: GCGGCCGCATTTTAATCACAAACCTATA

MAFF-F: CTCGAG TCCCACCCAGGAAAGGAGGA

MAFF-R: GCGGCCGC TCTTCTGTGCCAGGACGGAC

NFYC-F: CTCGAGTGAGCTGGCAAGGCCAAGGA

NFYC-R: GCGGCCGCTTGGGGGTAGCAGCTGTTCC

AREG-F: CTCGA GGATATCACATTGGAGTCACTG

AREG-R: GCGGCCGCAGCTGTAAAATAAATACA CTTC

CAMTA2-F: CTCGAG TACCTGCATCTTCCGCTGTG

CAMTA2-R: GCGGCCGC GCTGGTGCAGCGATGTTTAAT 
MAP2K1-F: CTCGAG TTGGGAAGCAACAAAGAGCG

MAP2K1-R: GCGGCCGC GGGGAAAGATAAATCCGACA

PCK2-F: CTCGAG GACTGCCAGGAGGCACAGAAGTCAT

PCK2-R: GCGGCCGCTCCTCCAACAATCTCATGAG

RTN4-F: CTCGAGAAACGCCCAAAATAATTAGTAGG

RTN4-R: GCGGCCGCGATACTTTGCTTTTACAGTTCACAA

FUT1-F: CTCGAGCAGCAGTACGTGGCTTCAGA

FUT1-R: GCGGCCGCTGGTTAGCAATGAGCACGGT

Supplemental Data 3, Mutagenesis oligonucleotides (5' - 3')

AREG-F: GAGTGTGTCGGAAGTCATAGCCATAAATGATGAGT CG

AREG-R: CTATGACTTCCGACACACTCCAATGTGATATCCTG.

MAP2K1: AGCCAGAGCCCTTGTGAGGGTTGATAGCTGGGGCT

MAP2K1: AGCCCCAGCTATCAACCCTCACAAGGGCTCTGGCT

AXL-F: CAGGATCCAAGCTAAGGTGTCGGTCTGGGGAAAACTCCAC

AXL-R: GTGGAGTTTTCCCCAGACCGACACCTTAGCTTGGATCCTG

FUT1-mut1-F: GGGGTGAGGGATGTGTCGGAAAATGGTACAGC

FUT1-mut1-R: GCTGTACCATTTTCCGACACATCCCTCACCCC

FUT1-mut2-F: CTTTTTTTTAAAGCTCATAATGTCGGAAGAGCTCCATATATTGGG

FUT1-mut2-R: CCCAATATATGGAGCTCTTCCGACATTATGAGCTTTAAAAAAAAG 
UM-SCC-23

Fadu
$2.08 \pm 0.54$

$0.88 \pm 0.30$
$1.60 \pm 0.57$

$7.05 \pm 2.69$

$0.75 \pm 0.33$
$6.14 \pm 2.29$ 


\begin{tabular}{lcccc} 
& 0h $(\%)$ & $6 h(\%)$ & $12 h(\%)$ & $24 h(\%)$ \\
\hline UM-SCC-23-con & $1.60 \pm 0.57$ & $0.73 \pm 0.05$ & $0.74 \pm 0.05$ & $0.88 \pm 0.09$ \\
UM-SCC-23-miR-34a & $7.05 \pm 0.47$ & $3.21 \pm 0.14$ & $2.27 \pm 0.15$ & $2.10 \pm 0.04$ \\
\hline
\end{tabular}


UM-SCC-23

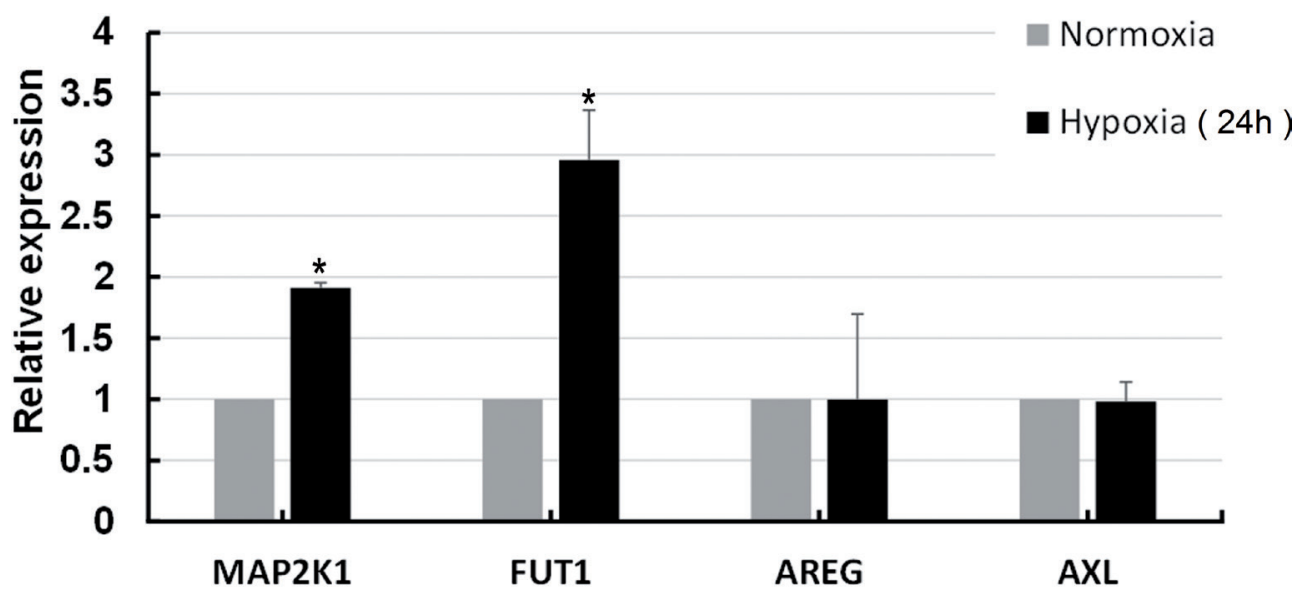

Fadu

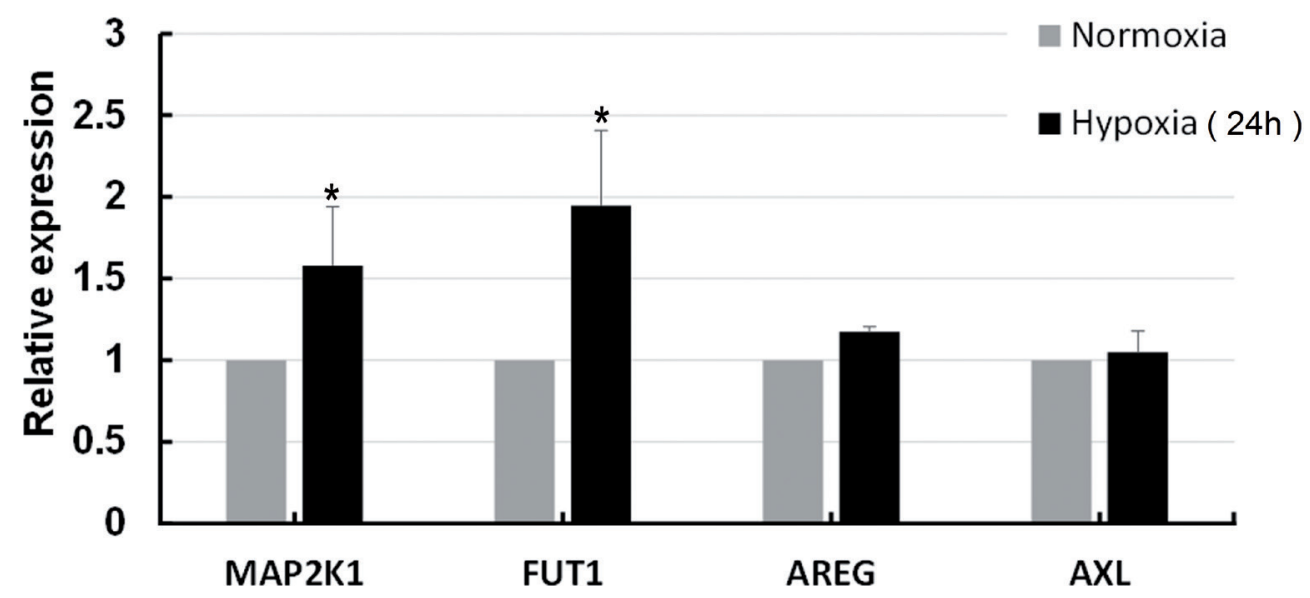

Supplemental data 6: the relative mRNA expression levels of AREG, AXL, MAPK2 and FUT1 in HNSCC cell lines induced by hypoxia. 